PROCEEDINGS OF THE

AMERICAN MATHEMATICAL SOCIETY

Volume 139, Number 12, December 2011, Pages 4513-4520

S 0002-9939(2011)10888-8

Article electronically published on April 4, 2011

\title{
LEGENDRIAN FRAMINGS FOR TWO-BRIDGE LINKS
}

\author{
SEBASTIAN BAADER AND MASAHARU ISHIKAWA
}

(Communicated by Daniel Ruberman)

\begin{abstract}
We define the Thurston-Bennequin polytope of a two-component link as the convex hull of all pairs of integers that arise as framings of a Legendrian representative. The main result of this paper is a description of the Thurston-Bennequin polytope for two-bridge links. As an application, we construct non-quasipositive surfaces in $\mathbb{R}^{3}$ all of whose sub-annuli are quasipositive.
\end{abstract}

\section{INTRODUCTION}

A Legendrian knot in the standard contact space $\mathbb{R}^{3}$ defines an integer, called a Thurston-Bennequin number, via its contact framing. The maximal ThurstonBennequin number of a knot is obtained by maximizing this integer over all Legendrian representatives of that knot. Precise values of the maximal ThurstonBennequin number are known for some special classes of knots, such as positive knots or two-bridge knots ([10, [5]). Given a link $L \subset \mathbb{R}^{3}$ with two components, we may ask which pairs of integers can be realised as framings of a Legendrian representative. The convex hull of these pairs of integers defines a polytope in $\mathbb{R}^{2}$, which we call the Thurston-Bennequin polytope $\Delta(L)$ of the link $L$. As an example, the Thurston-Bennequin polytope of the negative torus link of type $T(2,-4)$ is shown in Figure 1 (this is a special case of Theorem 1.1 below). In this paper we will

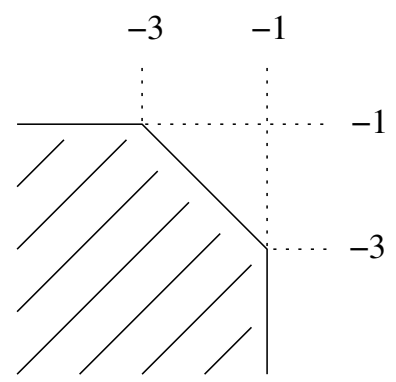

FiguRE 1. $\Delta(T(2,-4))$

determine the Thurston-Bennequin polytope for oriented two-bridge links. Our description makes use of the maximal Thurston-Bennequin number for oriented links,

Received by the editors October 26, 2009 and, in revised form, May 7, 2010 and October 11, 2010 .

2010 Mathematics Subject Classification. Primary 57M27.

(C)2011 American Mathematical Society 4513 
denoted by $\overline{t b}$, and the linking number $l k$. It is invariant under an overall change of orientation.

Theorem 1.1. Let $L$ be a two-bridge link with two components $L_{1}, L_{2}$. The Thurston-Bennequin polytope of $L$ is

$$
\Delta(L)=\left\{\left(x_{1}, x_{2}\right) \in \mathbb{R}^{2} \mid x_{1} \leq-1, x_{2} \leq-1, x_{1}+x_{2} \leq \overline{t b}(L)-2 l k(L)\right\} .
$$

Moreover, every integer point of $\Delta(L)$ is realised by a Legendrian framing.

Theorem 1.1 has an interesting application concerning quasipositive surfaces in $\mathbb{R}^{3}$. Quasipositive surfaces can be defined as the minimal family of embedded surfaces in $\mathbb{R}^{3}$ containing the positive Hopf band (i.e., the fibre of the positive Hopf link), which is closed under the plumbing operation and under taking incompressible sub-surfaces $([6], 7])$. Here a sub-surface is called incompressible if its embedding into the ambient surface induces an injective map on the level of fundamental groups. By definition, every incompressible sub-surface of a quasipositive surface is quasipositive. In particular, this is true for all incompressible sub-annuli. It is tempting to conjecture the converse: a surface embedded in $\mathbb{R}^{3}$ is quasipositive if all its incompressible sub-annuli are. In the case of pretzel surfaces, this is true [8]. In general, it is false.

Theorem 1.2. There exist non-quasipositive connected surfaces in $\mathbb{R}^{3}$ all of whose incompressible sub-annuli are quasipositive.

We will construct a surface with the above properties in the third section. The next section is devoted to the proof of Theorem 1.1, which is based on Ng's work [5].

\section{Construction of Legendrian Representatives}

The standard contact structure on $\mathbb{R}^{3}$ is induced by the 1 -form $d z-y d x$. As mentioned above, a Legendrian knot has a natural framing induced by the contact planes. The Thurston-Bennequin number is defined as the linking number of the link induced by that framing. In order to define the Thurston-Bennequin number for oriented links, it is convenient to introduce the front projection of links, i.e. the orthogonal projection to the $y$ - $z$-plane. Front projections of Legendrian links contain cusps, as shown in Figure 3. The Thurston-Bennequin number $t b(\mathcal{L})$ of an oriented Legendrian link $\mathcal{L} \subset \mathbb{R}^{3}$ is defined as

$$
t b(\mathcal{L})=w(D)-\frac{1}{2} c u(D),
$$

where $w(D)$ and $c u(D)$ denote the writhe (i.e., the algebraic crossing number) and the number of cusps of the front diagram $D$ of $\mathcal{L}$, respectively. For example, the Thurston-Bennequin number of the Legendrian link at the bottom of Figure 3 is -3 or -7 , depending on the choice of orientation. Let $\mathcal{L}=\mathcal{L}_{1} \cup \mathcal{L}_{2}$ be a Legendrian representative of a link $L$ with two components $L_{1}, L_{2}$. The following equality is an immediate consequence of the above definition:

$$
t b(\mathcal{L})=t b\left(\mathcal{L}_{1}\right)+t b\left(\mathcal{L}_{2}\right)+2 l k(L) .
$$

Therefore, if a pair of integers $\left(x_{1}, x_{2}\right)$ is induced by a Legendrian framing of $L$, then the following three inequalities hold:

$$
x_{1} \leq \overline{t b}\left(L_{1}\right), x_{2} \leq \overline{t b}\left(L_{2}\right), x_{1}+x_{2} \leq \overline{t b}(L)-2 l k(L) .
$$


Proof of Theorem 1.1. Let $L$ be a two-bridge link with components $L_{1}$ and $L_{2}$. Since these components are unknotted, we have

$$
\overline{t b}\left(L_{1}\right)=\overline{t b}\left(L_{2}\right)=-1 .
$$

Theorem 1.1 is settled once we have shown that every integer point on the diagonal line segment with endpoints $(-1, \overline{t b}(L)-2 l k(L)+1),(\overline{t b}(L)-2 l k(L)+1,-1)$ is realised by a Legendrian framing. Indeed, if a pair of integers $\left(x_{1}, x_{2}\right)$ is realised, then so are the pairs $\left(x_{1}-1, x_{2}\right)$ and $\left(x_{1}, x_{2}-1\right)$, by simple stabilisations. Here a stabilisation is a local operation that replaces an arc of a link component by a zig-zag, as shown in Figure 2. More precisely, this is a positive stabilisation (a negative stabilisation can be imagined by looking at the zig-zag on the left of Figure 4). Either stabilisation decreases the Thurston-Bennequin number of the link component with the zig-zag by 1 .

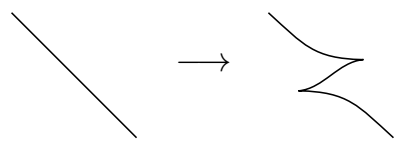

FiguRE 2

Two-bridge links were introduced and classified by Schubert 9]. An explicit description by finite continued fractions is due to Conway [3] (see also Murasugi's book [4, Chapter 9, for a thorough treatment of the subject). In [5], Ng proved that every two-bridge link has a diagram corresponding to an alternating continued fraction

$$
\left[a_{1},-a_{2}, \ldots,(-1)^{n-1} a_{n}\right]=a_{1}+\frac{1}{-a_{2}+\ldots+\frac{1}{(-1)^{n-1} a_{n}}}
$$

with $a_{i} \geq 2$, for all $i \in\{1, \ldots, n\}$. These diagrams in turn define a canonical front projection $F\left(a_{1}, \ldots, a_{n}\right)$, called a Legendrian rational front. There are two cases to be distinguished, according to whether $n$ is even or odd. These are illustrated by concrete examples, $F(3,3)$ and $F(2,3,4)$, in Figure 3. A regular diagram of the corresponding two-bridge links can be read off from the front by replacing each double point by a link crossing, taking the arc with the negative slope to be above.
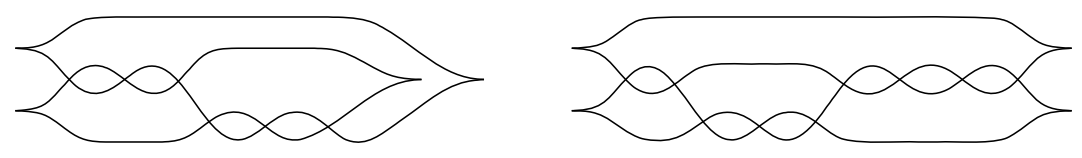

Figure $3 . F(3,3)$ and $F(2,3,4)$

Let $D=F\left(a_{1}, \ldots, a_{n}\right)$ be a rational front projection for a Legendrian representative $\mathcal{L}$ of the link $L$. According to $\mathrm{Ng}, D$ realises the maximal Thurston-Bennequin number of $L$. We observe that the upper component of $D$, say $\mathcal{L}_{1}$, has the ThurstonBennequin number -1 , so the corresponding point of the Thurston-Bennequin polytope has coordinates $(-1, \overline{t b}(L)-2 l k(L)+1)$. We will gradually move from this 
point along the diagonal line towards the point $(\overline{t b}(L)-2 l k(L)+1,-1)$ via a suitable family of Legendrian front diagrams.

We first assume that some of the coefficients $a_{i}$ corresponding to self-crossings of the lower component $\mathcal{L}_{2}$ are odd. In this case we will modify the continuous fraction and arrange all the numbers $a_{i}$ corresponding to self-crossings of $\mathcal{L}_{2}$ to be even. For this purpose we use the fact that the value of a continued fraction is preserved if we replace a segment of the form $a_{i},-a_{i+1}$ by the segment $a_{i}-1,1, a_{i+1}-1$ and change the signs of all subsequent coefficients, provided $a_{i}, a_{i+1} \geq 2$. This replacement can be done independently at all self-crossings (note that if the number $a_{i}$ corresponds to self-crossings of $\mathcal{L}_{2}$, then $a_{i+1}$ does not). However, this produces a non-alternating continued fraction: the crossings corresponding to coefficient 1 carry the wrong sign. We may still associate a front diagram to this fraction by introducing a zigzag to the component $\mathcal{L}_{2}$ at all single crossings with the wrong sign. Figure 4 shows the two possible ways of introducing a zig-zag at a crossing.
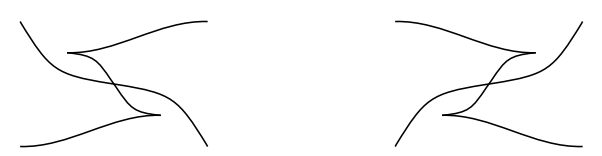

Figure 4. Two kinds of zig-zag

In this manner we obtain a front diagram $D^{\prime}$ which still maximises the ThurstonBennequin number of $L$. In fact all three numbers $t b(\mathcal{L}), t b\left(\mathcal{L}_{1}\right), t b\left(\mathcal{L}_{2}\right)$ are the same for both diagrams $D, D^{\prime}$. This follows from equality (2.1) and the fact that all selfcrossings of $\mathcal{L}_{2}$ are negative (the increase in the writhe from $D$ to $D^{\prime}$ equals the number of cancelled self-crossing of $\mathcal{L}_{2}$ and is therefore neutralised by the additional pairs of cusps).

Next we wish to modify $D^{\prime}$ so that the number of self-crossings of $\mathcal{L}_{2}$ between the lower two strands is at least as high as the number of self-crossings of $\mathcal{L}_{2}$ between the middle two strands. If this is not yet the case, we may simply turn the diagram $D^{\prime}$ upside down and drag the lower strand to the top; see Figure 5 for even and odd $n$, respectively. Again, this operation does not affect the numbers $t b(\mathcal{L}), t b\left(\mathcal{L}_{1}\right)$, $\operatorname{tb}\left(\mathcal{L}_{2}\right)$.

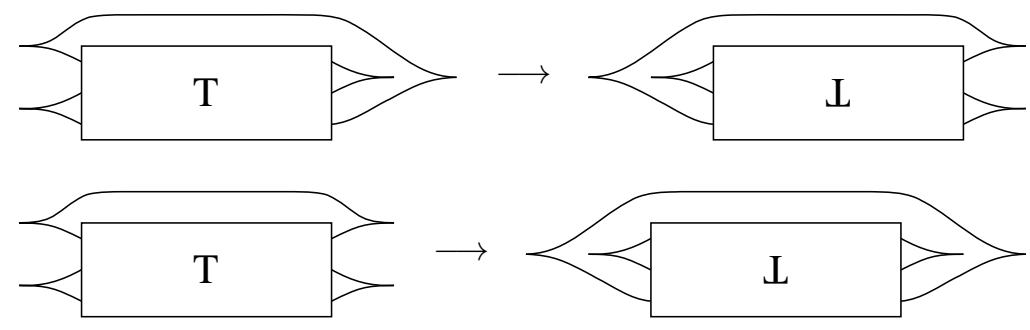

FiguRE 5

At last, we will describe two kinds of operations on the diagram $D^{\prime}$ that do change the numbers $t b\left(\mathcal{L}_{1}\right)$ and $t b\left(\mathcal{L}_{2}\right)$. 
(1) Replace a zig-zag on the component $\mathcal{L}_{2}$ by a zig-zag on the component $\mathcal{L}_{1}$ (compare Figure 4 ). This operation changes the Thurston-Bennequin numbers of $\mathcal{L}_{1}$ and $\mathcal{L}_{2}$ by -1 and +1 , respectively.

(2) Replace two consecutive self-crossings of $\mathcal{L}_{2}$ at the lower level by two consecutive self-crossings of $\mathcal{L}_{1}$ at the upper level, as shown in Figure 6. This operation corresponds to a double flype, but not to a Legendrian isotopy of front diagrams. In fact the Thurston-Bennequin numbers of $\mathcal{L}_{1}$ and $\mathcal{L}_{2}$ change by -2 and +2 , respectively.

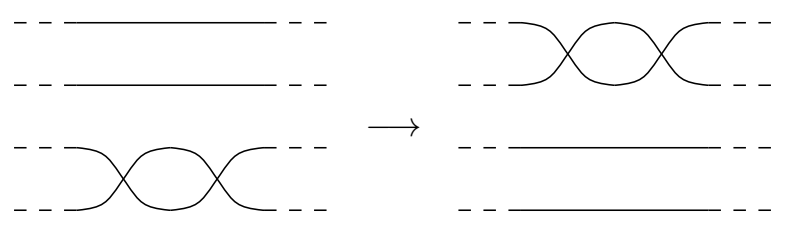

Figure 6

We will now explain how to realise all integer points up to the midpoint on the diagonal segment of the Thurston-Bennequin polytope of the link $L$. Let $p \in 2 \mathbb{N}$ be the total number of self-crossings of $\mathcal{L}_{2}$ after the above modifications, and let $q \geq 1$ be the number of zig-zags we introduced. We observe that the number of integer points on the diagonal segment is $p+q+1$. At least half of the self-crossings of $\mathcal{L}_{2}$ are on the lower two strands, say $2 r \geq \frac{p}{2}$. Thus we may apply up to $r$ operations of type (2) and $q$ operations of type (1). This allows us to step up to the midpoint of the diagonal, since $2 r+q>\frac{p+q}{2}$.

We finally discuss the case when all coefficients corresponding to self-crossings of $\mathcal{L}_{2}$ are even, as e.g. for the front $F(2,2,2,2,2,2,2)$. Let $a_{k}$ be the last coefficient corresponding to self-crossings of the component $\mathcal{L}_{2}$. As before, we may replace the segment $a_{k},-a_{k+1}$ of the continued fraction by the segment $a_{k}-1,1, a_{k+1}-1$ and introduce one zig-zag at the single crossing corresponding to the coefficient 1. Let $p \in 2 \mathbb{N}+1$ be the total number of self-crossings of $\mathcal{L}_{2}$ after this modification. Thus the number of integer points on the diagonal segment is $p+2$. As before, we may apply the operation of Figure 5, if necessary, and obtain a diagram where at least half of the self-crossings of $\mathcal{L}_{2}$ are on the lower two strands. Depending on whether $p=4 r-1$ or $p=4 r-3$, we can apply at least $r$ or $r-1$ operations of type (2), respectively. Moreover, we may apply one operation of type (1). In any case, this allows us to make at least $\frac{p+1}{2}$ steps up to the midpoint of the diagonal (if $p=4 r-1$, then $2 r+1>\frac{p+1}{2}$; if $p=4 r-3$, then $2(r-1)+1=\frac{p+1}{2}$ ).

For covering the other half of the diagonal, we remark that we could have started with a rational diagram where the upper component is $\mathcal{L}_{2}$ instead of $\mathcal{L}_{1}$.

It is natural to ask whether the Thurston-Bennequin polytope of a twocomponent link can always be described by three linear inequalities, and whether all its integer points are realised by Legendrian framings. We do not know the answer to these questions. However, there do exist links whose Thurston-Bennequin polytope has an even simpler shape: positive braid links. According to Bennequin [2] (see also [10]), every positive braid link has a natural Legendrian representative whose Thurston-Bennequin number is maximal and equals the number of crossings minus the number of strands of the braid. Moreover, the Thurston-Bennequin 
number of every single component of that representative is maximal. Thus the Thurston-Bennequin polytope of a two-component positive braid link is described by two inequalities only:

$$
x_{1} \leq \overline{t b}\left(L_{1}\right), \quad x_{2} \leq \overline{t b}\left(L_{2}\right)
$$

The same is evidently true for all two-component split links.

\section{Application Related to QUaSipositivity}

Originally, a surface embedded in $\mathbb{R}^{3}$ was called quasipositive if it was isotopic to a certain positively braided surface. Rudolph's characterisation results say that quasipositivity of surfaces is inherited by incompressible sub-surfaces and is preserved under the plumbing operation ([6], [7]). Moreover, every quasipositive surface can be embedded incompressibly on the fibre surface of a torus link. The latter are plumbings of finitely many positive Hopf bands. The following definition is therefore justified.

Definition 3.1. A surface embedded in $\mathbb{R}^{3}$ is called quasipositive if it is obtained from a disc by plumbing finitely many positive Hopf bands and passing to an incompressible sub-surface.

An alternative definition for quasipositivity was recently formulated in the setting of contact geometry [1: quasipositive surfaces are precisely the Legendrian ribbons in the standard contact $\mathbb{R}^{3}$. For our purposes, it suffices to know that every Legendrian link defines a Legendrian ribbon by replacing its components by disjoint annuli with the obvious framings.

Proof of Theorem 1.2. We will construct an embedded surface, starting from the Legendrian ribbon associated to the front projection shown in Figure 7. This front realises the maximal Thurston-Bennequin number of the corresponding link (the negative torus link of type $(2,-4))$, by $\mathrm{Ng}[5$. Therefore, if we remove the full twist encircled in Figure 7, we obtain a surface, say $F$, which is not a Legendrian ribbon, hence a non-quasipositive surface. Nevertheless, both incompressible sub-annuli of $F$ are obviously quasipositive.
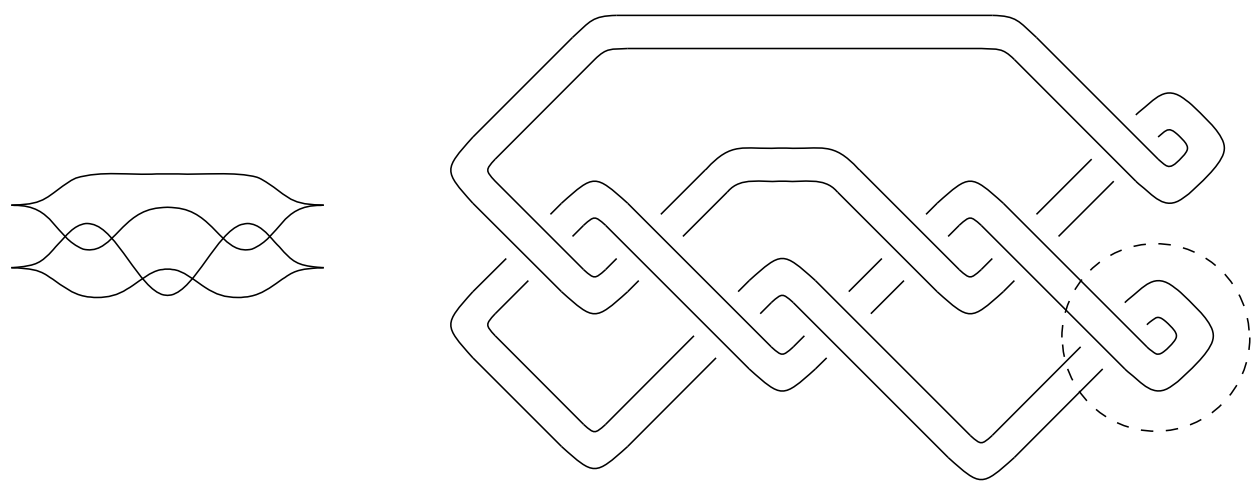

FiguRE 7 
Connecting these two sub-annuli by a small band, as shown at the top of Figure 8 , we obtain a connected surface $F^{\prime}$, which is again non-quasipositive, since it contains $F$ as an incompressible sub-surface. We claim that all incompressible sub-annuli of $F^{\prime}$ are quasipositive. Every incompressible sub-annulus of $F^{\prime}$ is parallel to the boundary of $F^{\prime}$. Indeed, the topological type of the surface $F^{\prime}$ is a pair of pants; thus the core curve of an incompressible sub-annulus divides $F^{\prime}$ into a pair of pants and an annulus. We already checked the quasipositivity for two sub-annuli of $F^{\prime}$. The third sub-annulus is shown at the bottom of Figure 8 . It is manifestly a Legendrian ribbon, hence quasipositive.

The above construction works with other two-bridge links as well, for example with all negative torus links of type $L(2,-2 n), n \geq 2$.
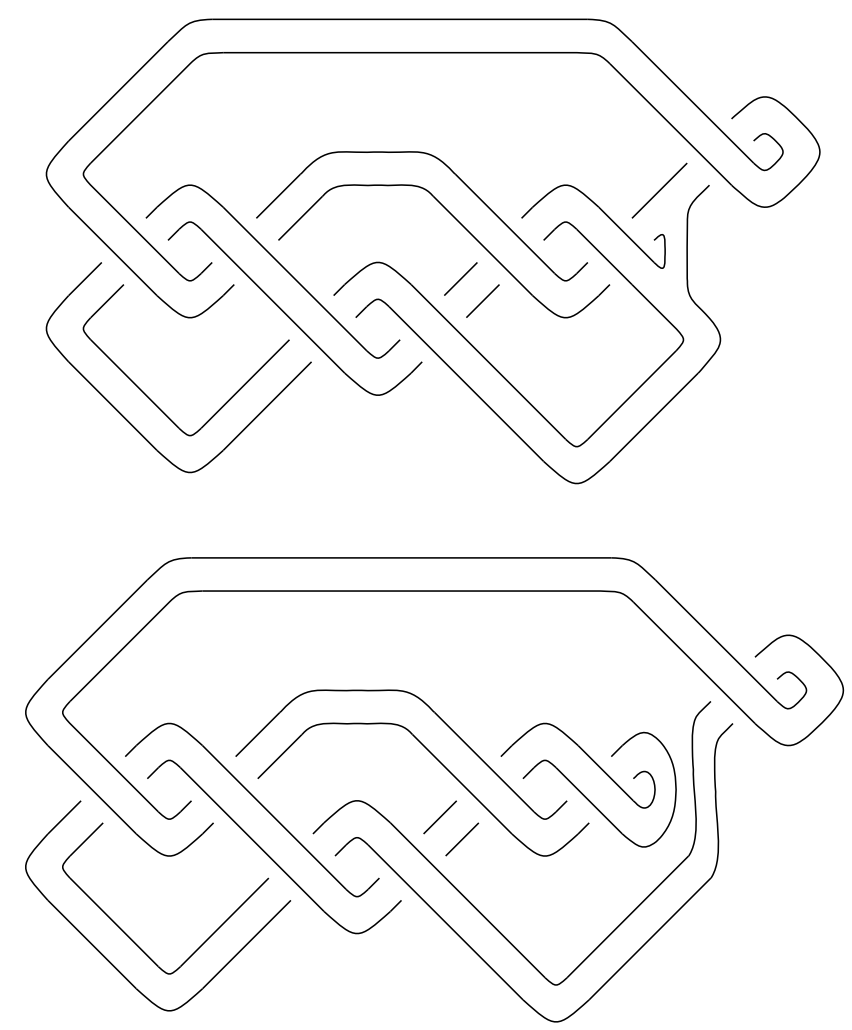

Figure 8

\section{ACKNOWLEDGEMENTS}

This work was done while the first author visited the Mathematical Institute at Tohoku University in July 2009. He would like to thank the members of the Institute for their hospitality and generous support. The second author is supported by MEXT, Grant-in-Aid for Young Scientists (B) (No. 22740032). Both authors would like to thank the referee, whose comments led to a great clarification of the proof of Theorem 1.1. 


\section{REFERENCES}

1. S. Baader, M. Ishikawa: Legendrian graphs and quasipositive diagrams, Ann. Fac. Sci. Toulouse Math. 18 (2009), 285-305. MR2562830

2. D. Bennequin: Entrelacements et équations de Pfaff, Astérisque 107-108 (1983), 87-161. MR7753131 (86e:58070)

3. J. H. Conway: An enumeration of knots and links, and some of their algebraic properties, Computational Problems in Abstract Algebra (Proc. Conf., Oxford, 1967), 329-358, Pergamon, Oxford. MR0258014 (41:2661)

4. K. Murasugi: Knot theory and its applications, Birkhäuser Boston, 1996. MR.1391727 (97g:57011)

5. L. L. Ng: Maximal Thurston-Bennequin number of two-bridge links, Algebr. Geom. Topol. 1 (2001), 427-434. MR1852765 (2002e:57020)

6. L. Rudolph: Constructions of quasipositive knots and links. III. A characterization of quasipositive Seifert surfaces, Topology 31 (1992), no. 2, 231-237. MR1167166 (93g:57014)

7. L. Rudolph: Quasipositive plumbing (constructions of quasipositive knots and links. V), Proc. Amer. Math. Soc. 126 (1998), no. 1, 257-267. MR1452826 (98h:57024)

8. L. Rudolph: Quasipositive pretzels, Topology Appl. 115 (2001), no. 1, 115-123. MR1840734 (2003a:57016)

9. H. Schubert: Knoten und Vollringe, Acta Math. 90 (1953), 131-286. MR0072482 (17:291d)

10. T. Tanaka: Maximal Bennequin numbers and Kauffman polynomials of positive links, Proc. Amer. Math. Soc. 127 (1999), no. 11, 3427-3432. MR.1616601 (2000b:57014)

Mathematisches Institut, Universität Bern, Sidlerstrasse 5, CH-3012 Bern, SwitzerLAND

E-mail address: sebastian.baader@math.unibe.ch

Mathematical Institute, Tohoku University, Sendai, 980-8578, Japan

E-mail address: ishikawa@math.tohoku.ac.jp 